\title{
Development and application of a compartmental model for estimating digesta passage in horses
}

\author{
C Corino, F Fontana, N Miraglia, P Zanetti \\ Università degli Studi del Molise \\ Dipartimento di Scienze Animali, Vegetali e dell'Ambiente, Via Cavour 50, \\ Campobasso, Italy
}

Three heavy-breed dry mares, 3 light horses and 3 heavy breed mares in late pregnancy and early lactation were monitored using a single dose of $\mathrm{Cr}$-mordanted hay. Total faeces were collected at increasing time intervals (Miraglia et al, 1992). The results of this investigation let it be supposed that marker excretion patterns in the faeces could be described by a 2-exponential components model. The application of the 2- compartmental model to ruminants (Grovum et Williams, 1973) did not give satisfactory statistical results. Thus, a new approach had to be found.

The improvement with respect to the previous approach lies in the assumption that some interactions exist between the 2 compartments. It was surmised that they interact through a feedback mechanism. From the mathematical point of view this corresponds to combining the effects of the 2 compartments in a non-trivial manner. A possible combination of the functions corresponding to the 2 volumes is the so-called master equation (Prigogine, 1961): $y(t)=y_{1}(t) \cdot(1-y(t))+y_{2}(t) \cdot y(t)$ where the 2 equations $y_{1}(t)$ and $y_{2}(t)$ are: $y_{1}(t)=1-\exp \left(-t k_{1}\right) ; y_{2}(t)=1-\exp (-t)$ $k_{2}$ ) where: $y(t)$ is the percentage of marker collected (cumulative amount), $t$ is the time, $k_{1}$ and $k_{2}$ are the rate constants for the 2 compartments. The following assumptions hold: a) the rate of passage can be considered a nonlinear dissipative process; b) it may be described by 2 concomitant global rate processes; $c$ ) both the processes fulfill detailed stationary conditions. As far as the fitting procedure is concerned, 2 considerations lead to the use of "cumulative data" instead of the experimental data in their rough form. First of all, the sampling intervals are not equally spaced. This problem is simple to overcome, but could induce some problems in the fitting procedure. The amount of marker recovered in a given interval is the overall quantity issued in the interval. Thus it ought to be equally distributed over the total time lag. In this way the hypothesis of constant emission is assumed.

This 2-compartmental model was applied to the experimental data previously obtained on horses (Miraglia et al, 1992). The usual statistical tests have shown that the model works to a good level of accuracy (in any case $>90 \%$ ).

Miraglia N, Poncet C, Martin-Rosset W (1992) Ann Zootech (41) (1), 69

Grovum WL, Williams VJ (1973) Br J Nutr 30 , 313-329

Prigogine $L$ (1961) Thermodynamics of irreversible Processes. Flamingo, NY 